\title{
Prevalence of Extended-Spectrum $\beta$-Lactamase- Production among Clinical Gram-Negative Bacilli Isolates in A Tertiary Hospital, Southwest Nigeria
}

\author{
Oluwaseun E. Adewole and Babatunde W. Odetoyin
}

\section{ABSTRACT}

Extended spectrum $\beta$-lactamases (ESBLs) have emerged as important mechanisms of antimicrobial resistance to beta-lactam drugs in hospitals, thereby limiting the available treatment options for infections caused by these microorganisms. The objectives of this study were to determine the prevalence of ESBL production among clinical isolates of Gram-negative bacilli from Obafemi Awolowo University Teaching Hospitals Complex, IleIfe, Nigeria as part of the global efforts to provide information for the containment of the spread of antimicrobial resistance. This is a laboratorybased study of 186 consecutive non-duplicate Gram-negative bacilli (GNB) isolated from diverse clinical samples at the Microbiology laboratory of the hospital after standard aerobic cultures. The isolates were identified by conventional biochemical test scheme and commercial GNB API 20E identification kit. Antimicrobial susceptibility testing (AST) of each isolate was determined by the disk diffusion technique. Isolates presumptively identified as ESBL producers from the AST assay were confirmed by the combination disks method. Escherichia coli $(n=43 ; 23.1 \%)$ predominated among the organisms, followed by Enterobacter $\operatorname{spp}(\mathrm{n}=23 ; 12.4 \%)$ and Klebsiella spp ( $\mathrm{n}=22 ; 11.8 \%)$. Most of the organisms $(95.7 \%)$ were resistant to tetracycline and ampicillin, but the least resistance $(4.3 \%)$ was exhibited to imipenem. Fifty-eight $(31.2 \%)$ isolates were ESBL producers with a preponderance of $E$. coli $(n=15 ; 25.9 \%)$. The ESBL producers were commonly isolated from urine samples $(n=31 ; 53.4 \%)$, and were significantly more resistant to levofloxacin, ciprofloxacin, nalidixic acid, gentamicin, augmentin, cefepime, ceftriaxone, ceftazidime and cefotaxime $(p<0.05)$. This study has highlighted the therapeutic implications of the presence of EBSL in clinical isolates which was depicted by their multi-drug resistance phenotypes, thus emphasizing the need for continuous surveillance of bacterial resistance, institution of antimicrobial resistance stewardship in our hospital and regular screening of ESBLs in clinical isolates to prevent treatment failure.

Keywords: Clinical isolates, esbl, Gram negative bacilli, multi-drug resistance.

\section{INTRODUCTION}

Gram-negative bacilli have not relented from being one of the important causes of infections which result in high morbidity and mortality in developing countries [1]. Antimicrobial resistance in Gram-negative bacilli has been on the increase in the last few decades particularly as a result of $\beta$-lactamases production, especially extended-spectrum betalactamases (ESBLs) which is a common resistance mechanism of Gram-negative bacilli [2]-[4]. The ESBL confers resistance to all beta-lactams except cephamycins and carbapenems. ESBL genes are often located on plasmids which can be transferred from one strain to another and between bacterial species [4].

ESBLs have become an increasingly significant resistance mechanisms among Enterobacteriaceae in different parts of the world [5]. Infections by ESBLs producing organisms are
Submitted : August 24, 2021

Published : September 21, 2021

ISSN: $2593-8339$

DOI: $10.24018 /$ ejmed.2021.3.5.1031

O. E. Adewole

University of Ibadan, Oyo State, Nigeria.

(e-mail: oeadewoler@yahoo.com)

B.W. Odetoyin*

Obafemi Awolowo University, Ile-Ife, Osun State, Nigeria.

(e-mail: odetoyin@yahoo.com)

*Corresponding Author associated with high cost, extended hospital stay and high mortality rate [6]. To worsen this matter, most clinical laboratories in developing countries do not routinely screen for ESBL-producers. This has resulted in avertable treatment failures in patients and outbreaks of resistant Gram-negative bacterial infections that require expensive control efforts [7], [8]. Inadequate and ineffective empirical antibiotic therapy has led to an increase in mortality. Therefore, early recognition and treatment are very important to prevent the dissemination of infections caused by ESBLs-producers [9], [10]. This study determined the prevalence of ESBL production among clinical isolates of Gram-negative bacilli from OAUTHC, Ile-Ife, Nigeria. 


\section{MAterials AND Methods}

This laboratory-based study was conducted at the Medical Microbiology and Parasitology laboratory of the Obafemi Awolowo University (OAU), Ile-Ife, Osun State, Nigeria.

A total of 186 non-repetitive Gram-negative bacilli were isolated from various clinical samples including blood, urine, stool, sputum and wound of infected patients from both outpatient and in-patient departments/units of the hospitals following aerobic cultures on MacConkey and Blood agar plates (Oxoid, Basingstoke, England) at $37^{\circ} \mathrm{C}$ for 24 hours. The isolates were identified to species level using conventional biochemical test scheme and the analytical profile index (API) 20E commercial kit (Bio Merieux, Germany). The identification was done with the aid of the Analytical profile index and ABS online identification software (www.tgw1916.net). The kit was validated with Escherichia coli ATCC 25922 (positive control) and sterile distilled water (negative control). The identified Gram negative bacilli were stored in tryptone soya agar prepared in cryovials and kept at $4{ }^{\circ} \mathrm{C}$ in a refrigerator for further examination.

The antimicrobial susceptibility of each isolate to selected antimicrobial agents was determined by the Kirby-Bauer disk diffusion method following the Clinical and Laboratory Standard Institute (CLSI) guidelines [11]. The antibiotic disks used were; gentamicin(10 $\mu \mathrm{g})$, ampicillin(10 $\mu \mathrm{g})$, augmentin $(30 \mu \mathrm{g})$, tetracycline $(30 \mu \mathrm{g})$, ciprofloxacin $(5 \mu \mathrm{g})$, levofloxacin $(5 \mu \mathrm{g})$, imipenem $(10 \mu \mathrm{g})$, chloramphenicol $(30 \mu \mathrm{g})$, nalidixic acid $(30 \mu \mathrm{g})$, streptomycin $(10 \mu \mathrm{g})$, trimethoprim $(5 \mu \mathrm{g})$, sulfonamide $(300 \mu \mathrm{g})$, ceftazidime $(30 \mu \mathrm{g})$, cefotaxime $(30 \mu \mathrm{g})$, cefepime $(30 \mu \mathrm{g})$, ceftriaxone $(30 \mu \mathrm{g})$, cefotaxime-clavulanate $(30 \mu \mathrm{g} / 10 \mu \mathrm{g})$, ceftazidimeclavulanate $(10 \mu \mathrm{g} / 1 \mu \mathrm{g})$ (Mast, England).

Antimicrobial disks were placed onto the surface of the agar by a disc dispenser (Remel, USA). After a few minutes, the plates were turned upside down and incubated at $37^{\circ} \mathrm{C}$ for $24 \mathrm{~h}$. After overnight incubation, the diameters of the inhibition zones were measured to the nearest millimetre, and isolates were categorised as susceptible, intermediate, or resistant using CLSI-specified interpretative criteria. For analysis, resistant and intermediate strains were grouped together in the resistant group. Escherichia coli ATCC 25922 was used as a reference strain for the test. Multidrug resistance (MDR) was defined as non-susceptibility of an isolate to at least one antimicrobial agent in three or more categories [12].

Isolates with inhibition zone diameters of $\leq 22 \mathrm{~mm}$ with ceftazidime $(30 \mu \mathrm{g})$ and/or $\leq 27 \mathrm{~mm}$ with cefotaxime $(30 \mu \mathrm{g})$ in the AST assay were identified as possible ESBL-producer and were selected for confirmation testing using a combination disk test as recommended by CLSI [11]. ESBL production was confirmed using the combination disk method. Cefotaxime $(30 \mu \mathrm{g})$, Ceftazidime $(30 \mu \mathrm{g})$ and cefotaxime $(30 \mu \mathrm{g})+$ clavulanic acid $(30 \mu \mathrm{g} / 10 \mu \mathrm{g})$, ceftazidime + clavulanic acid $(30 \mu \mathrm{g} / 10 \mu \mathrm{g})$ disks were placed at a suitable distance on Mueller Hinton agar plate that has been inoculated with standardized inoculum of each bacterial isolate and the plates were incubated overnight (18-24 h) at $37^{\circ} \mathrm{C}$. An increase of $>5 \mathrm{~mm}$ in the inhibition zone diameter for the combined disks (cefotaxime + clavulanic acid or ceftazidime + clavulanic acid) over the cefotaxime or ceftazidime single disk was confirmed the isolate as ESBL producer. Escherichia coli ATCC 25922 (ESBL-negative) and $K$. pneumoniae ATCC 700603 (ESBL positive) served as negative and positive control strains respectively.

\section{DATA ANALYSIS}

Qualitative data were analyzed with $\mathrm{R}$ statistical software package version 3.4 [13], and results presented in tables and graphs. Association between categorical variables was determined by the Chi-square test. Antimicrobial resistance (AMR) was analyzed by the AMR package of Berends et al. [14], in $\mathrm{R}$ statistical software. A p-value $<0.05$ was considered statistically significant.

\section{Results}

One hundred and eighty-six consecutive non-repetitive Gram-negative bacilli isolates were recovered from different clinical samples of a total of 173 patients. These include; urine $(n=78 ; 41.9 \%)$, stool $(n=2 ; 14.5 \%)$, blood $(n=27$; $14.5 \%)$, wound $(n=24 ; 12.9 \%)$, sputum $(n=16 ; 8.6 \%)$, high vagina swab $(n=5 ; 2.7 \%)$, endocervical swab $(n=3 ; 1.6)$ and aspirate $(n=4 ; 2.2 \%)$, with 13 samples yielding multiple isolates. Most isolates were recovered from in patients $(\mathrm{n}=140 ; 75.3 \%)$ while others $(\mathrm{n}=46 ; 24.7 \%)$ were recovered from outpatients (Table I). Escherichia coli $(n=43 ; 23.1 \%)$ was the most frequent bacteria pathogen recovered from the clinical samples, followed by Enterobacter spp $(\mathrm{n}=23$; $12.4 \%)$, Klebsiella spp $(\mathrm{n}=22 ; 11.8 \%)$, Pseudomonas $\mathrm{spp}$ $(\mathrm{n}=18 ; 9.7 \%)$, Raoutella terrigena $(\mathrm{n}=15 ; 8.1 \%)$, P. mirabilis $(\mathrm{n}=12 ; 6.5 \%)$, Serratia $\operatorname{spp}(\mathrm{n}=11 ; 5.9 \%)$ and Acinetobacter baumannii $(\mathrm{n}=8 ; 4.3 \%)$ (Fig. 1).

TABLE I: DISTRIBUTION OF GRAM NEGATIVE BACILLI ISOLATES WITH RESPECT TO CLINICAL SAMPLES OF PATIENTS

\begin{tabular}{ccc}
\hline \hline Parameters & $\begin{array}{c}\text { No (\%) of patients } \\
\text { with isolates (N=173) }\end{array}$ & $\begin{array}{c}\text { No (\%) of Gram- } \\
\text { negative bacilli } \\
\text { isolates }(\mathrm{N}=186)\end{array}$ \\
\hline \hline Type of patients & $129(74.6)$ & $140(75.3)$ \\
In patients & $44(25.4)$ & $46(24.7)$ \\
Outpatients & & \\
Type of clinical & $4(2.3)$ & \\
specimens & $26(15)$ & $4(2.2)$ \\
Aspirate & $3(1.7)$ & $3(14.5)$ \\
Blood & $5(2.9)$ & $5(2.7)$ \\
Endocervical swab & $2(1.2)$ & $2(1.1)$ \\
High vaginal swab & $13(7.5)$ & $16(8.6)$ \\
Semen & $25(14.5)$ & $27(14.5)$ \\
Sputum & $75(43.4)$ & $28(41.9)$ \\
Stool & $20(11.6)$ & $2(12.9)$ \\
Urine & & \\
Wound & &
\end{tabular}

The majority of the isolates were commonly resistant to ampicillin $(95.7 \%)$, tetracycline $(95.7 \%)$, sulphonamide (91.9\%), trimethoprim (90.3\%), augmentin (87.1\%), streptomycin $(83.3 \%)$, nalidixic acid $(76.9 \%)$, chloramphenicol (70.4\%), ciprofloxacin (55.9\%) and cefepime $(51.1 \%)$, but the least resistance was exhibited to imipenem (4.3\%) (Fig. 2). 


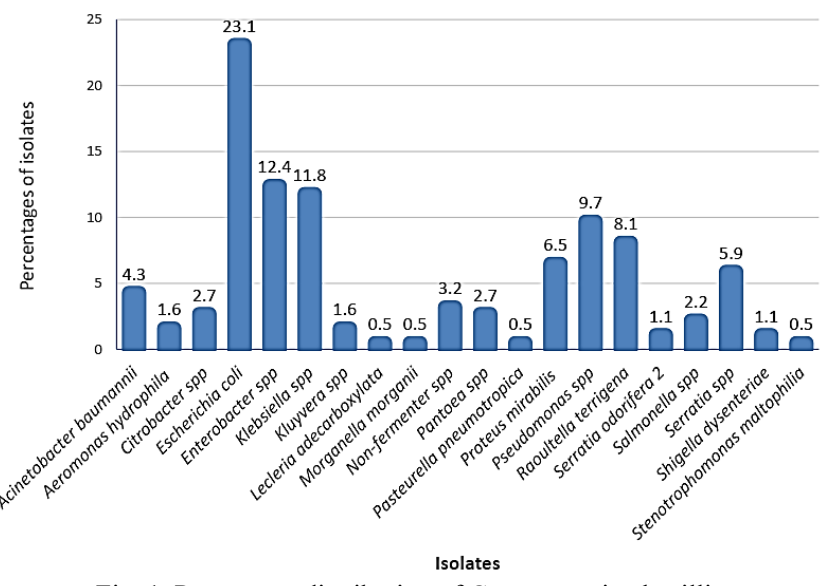

Fig. 1. Percentage distribution of Gram-negative bacilli.

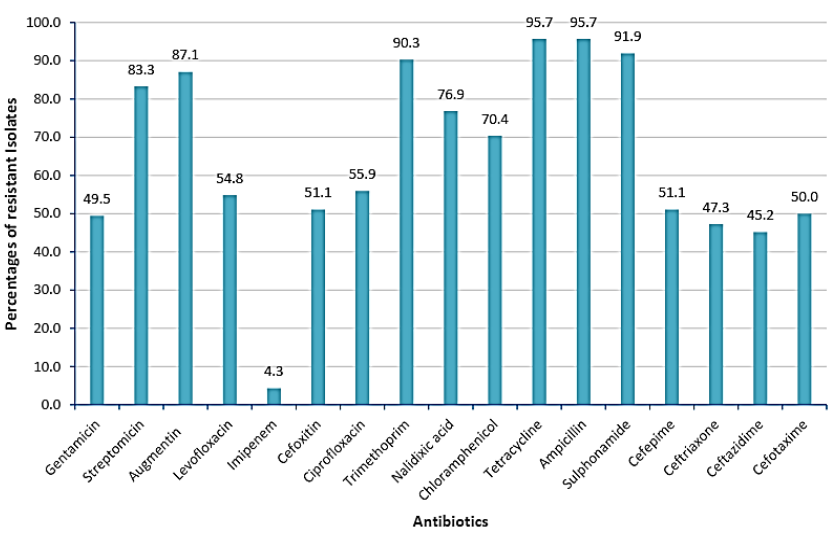

Fig. 2. Resistance profile of Gram-negative bacilli.

Of the 186 isolates, $58(31.2 \%)$ were ESBL producers. The most frequent ESBL producing bacteria was Escherichia coli $(\mathrm{n}=15: 25.9 \%)$, followed by Enterobacter $\mathrm{spp} .(\mathrm{n}=8 ; 14.0 \%)$, Klebsiella spp $(\mathrm{n}=7 ; 12.0 \%)$, Pseudomonas $\mathrm{spp}(\mathrm{n}=6 ; 10.0 \%)$ and Serratia spp $(\mathrm{n}=6 ; 10.0 \%)$ (Fig. 3). Most ESBL producing isolates were obtained from urine $(n=31 ; 53.4 \%)$, followed by blood $(n=9 ; 15.5 \%)$, wound $(n=6 ; 10.3 \%)$, stool $(n=6 ; 10.3 \%)$ and sputum $(n=4 ; 6.9 \%)$ (Fig. 4).

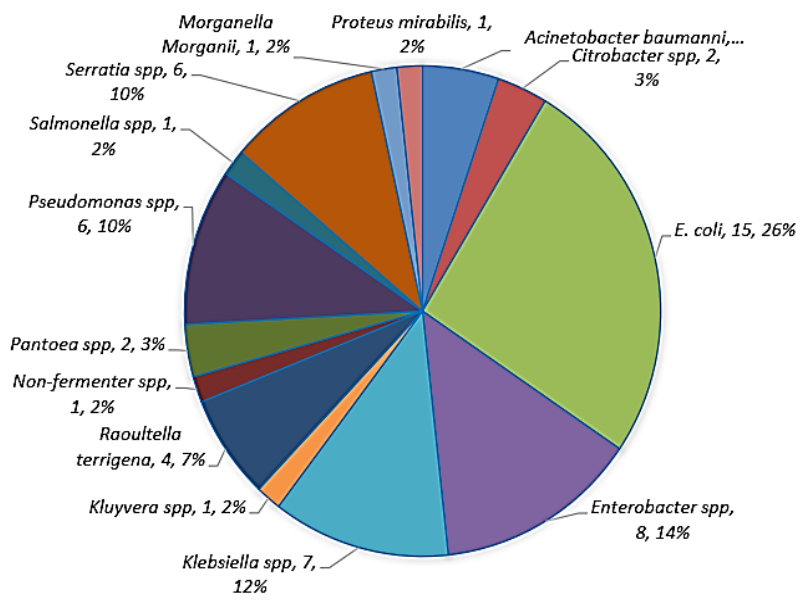

Fig. 3. Distribution of ESBL producing Gram-Negative bacilli $(\mathrm{n}=58)$.

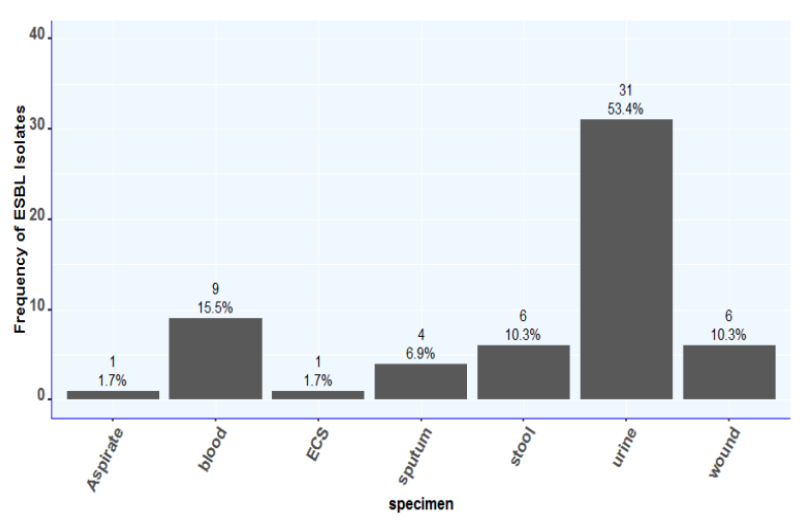

Fig. 4. Distribution of ESBL isolates in different specimens.

The ESBLs producers were significantly more resistant to augmentin, levofloxacin, gentamicin, ciprofloxacin, nalidixic acid, cefepime, ceftriaxone, ceftazidime and cefotaxime when compared with the non-ESBLs producers $(\mathrm{p}<0.05)$. Also, 4 (6.9\%) ESBLs producers and 3 (2.3\%) non-ESBLs producers showed resistance to imipenem (Table II). Fiftysix (96.6\%) ESBL producers were MDR while 118 (92.2\%) non-ESBL producers were MDR. There was no significant difference in the frequency of MDR between ESBL and nonESBL producers $(\mathrm{P}>0.05)$.

TABLE II: RESISTANCE PROFILE OF ESBL AND NON ESBL PRODUCING GRAM-NEGATIVE BACILLI

\begin{tabular}{cccc}
\hline \hline & $\begin{array}{c}\text { ESBL } \\
\text { Producers } \\
\mathrm{n}=58(\%)\end{array}$ & $\begin{array}{c}\text { Non ESBL } \\
\text { Producers } \\
\mathrm{n}=128(\%)\end{array}$ & $\mathrm{p}$ value \\
\hline \hline Gentamicin & $36(62.1)$ & $51 / 125(40.8)$ & $0.007^{*}$ \\
Streptomycin & $48(82.8)$ & $96 / 124(77.2)$ & 0.409 \\
Augmentin & $53(91.4)$ & $100(78.1)$ & $0.028^{*}$ \\
Levofloxacin & $37(63.8)$ & $58(45.3)$ & $0.020^{*}$ \\
Imipenem & $4(6.9)$ & $3(2.3)$ & 0.131 \\
Cefoxitin & $25(43.1)$ & $65(50.8)$ & 0.332 \\
Ciprofloxacin & $41(70.7)$ & $56(43.8)$ & $0.001^{*}$ \\
Trimethoprim & $51 / 57(89.5)$ & $105 / 125(84.0)$ & 0.328 \\
Nalidixic acid & $50(86.21)$ & $85 / 125(68.0)$ & $0.009^{*}$ \\
Chloramphenicol & $43(74.1)$ & $82 / 126(65.1)$ & 0.221 \\
Tetracycline & $53(91.4)$ & $113(88.3)$ & 0.528 \\
Ampicillin & $55(94.8)$ & $112 / 127(88.2)$ & 0.158 \\
Sulphonamide & $50(86.2)$ & $112 / 126(88.9)$ & 0.620 \\
Cefepime & $38 / 57(66.7)$ & $53(41.4)$ & $0.002^{*}$ \\
Ceftriaxone & $40 / 49(81.6)$ & $43 / 109(39.5)$ & $0.000^{*}$ \\
Ceftazidime & $52 / 54(92.9)$ & $60 / 109(55.1)$ & $0.000^{*}$ \\
Cefotaxime & $44(75.9)$ & $45 / 126(35.7)$ & $0.000^{*}$ \\
Multi drug & $56(96.6)$ & $118(92.2)$ & 0.325 \\
Resistance & & & \\
\hline \hline p $<0.05$. & & & \\
\hline
\end{tabular}

\section{Discussion}

In this study, E. coli $(24.3 \%)$ was the most frequent bacterium among the GNB recovered from samples of patients with suspected clinical infections in our study, which is similar to what have been previously reported by other investigators [15], [16]. The high frequency of $E$. coli isolation indicates its continued significance in the aetiology of urinary tract infections, gastrointestinal infections and bloodstream infections, which accounted for more than $85 \%$ of suspected clinical infections in this study. Majority of the Gram-negative isolates were from urine samples, which formed the highest number of clinical samples in this study, supporting the fact that urine is the most common routine samples received at the clinical microbiology laboratory, even as E. coli is the most common bacteria aetiology of 
uncomplicated urinary tract infection [17].

Antimicrobial resistance in the health care setting, although not a new problem, is worrisome as it affects the efficacy of antimicrobial agents. This problem is exacerbated in developing countries where several factors favour the emergence and spread of antimicrobial resistance [2]. The majority of the Gram-negative bacteria isolated in our study, were predominantly resistant to ampicillin (95.7\%), tetracycline $(95.7 \%)$, sulphonamide $(91.9 \%)$, and trimethoprim $(90.3 \%)$. The organisms were also resistant to streptomycin, chloramphenicol, ciprofloxacin, gentamicin, and third-generation cephalosporins. Increased resistance of Gram-negative bacteria to these antibiotics has also been reported by other investigators. These findings highlight the negative repercussions of Nigeria's lax and unenforced antibiotic prescribing regulations in the context of a high infectious disease burden. Antibiotics are frequently used and abused giving resistant organisms a competitive advantage [18].

In our study, 58 (31.2\%) GNB were ESBL producers and were obtained from different clinical specimens. The reported incidence of ESBL-producing organisms varies between 10 and $55 \%$ from one region to another [19]-[21]. A recent review of the prevalence of ESBL-producing Enterobacteriaceae in Nigerian hospitals by Musa et al. reported a prevalence of $34.6 \%$ [22]. The high incidence of ESBLs found in our study could be attributed to the frequent use and misuse of third-generation cephalosporins [23]. In several parts of the world, ESBLs are becoming an increasingly prominent resistance mechanism among Enterobacteriaceae [24]. Clinical infections caused by ESBL producing bacteria are associated with high costs, extended hospital stay and a high mortality rate [25]. Consequently, proper monitoring of the prevalence and type of ESBL in clinical isolates may aid in defining apposite therapeutic options.

The majority of ESBL-producing bacteria were recovered from urine (53.4\%), followed by blood (15.5\%) and wound $(10.3 \%)$, which is consistent with findings from prior research in which urine was the primary source of ESBLproducing bacteria [26]. However, in other research, blood was the primary source of ESBL-producers [27], [28]. The disparity could be attributed to differences in research participants, risk factors, or the level of antibiotic use.

The commonest ESBLs producing organisms isolated were E. coli 15 (25.9\%), followed by Enterobacter spp 11 (19\%). This is consistent with the findings of Teklu et al. [29], Giwa et al. [7] and Mohammed et al., [30], who reported that more E. coli than other Gram-negative bacilli produced ESBL. ESBLs producers were significantly more resistant to augmentin, levofloxacin, gentamicin, ciprofloxacin, nalidixic acid, cefepime, ceftriaxone, ceftazidime and cefotaxime when compared with non- ESBLs producers. The observed trend of resistance to most tested antibiotics could be related to misuse of these antimicrobial agents in our country, easy availability of these antibiotics, absence of prescription and a lack of antibiotic policies in Nigeria [18]. In Nigeria, the detection of ESBL producers is not routinely conducted due to lack of awareness, limited resources and facilities in most laboratories. The presence of considerable antibiotic resistance among ESBL producers emphasizes the urgent necessity to incorporate detection of ESBL producers into standard laboratory techniques.

Most of the GNB (96.2\%) were sensitive to imipenem. This is comparable to the work of Ogefere et al. [31] who reported that imipenem was the most active antimicrobial agent against ESBL-producing and non-ESBL-producing Gram-negative bacilli. Despite the fact that imipenem was only recently introduced, it was found to be resistant in $6.9 \%$ and $2.3 \%$ of ESBL-producers and non-ESBL-producers, respectively, in this study. As a result, the treatment choices for infections caused by these organisms are severely limited. It is possible that the isolates that were resistant to imipenem have plasmids encoding carbapenemase, an enzyme that hydrolyzes carbapenems, the class to which imipenem belongs. A previous study by Codjoe and Donkor, [32] linked the therapeutic failure of imipenem to multiple betalactamases production. The occurrence of imipenem resistance among the non-ESBL producers suggests the involvement of alternative resistance mechanisms like mutation, efflux pump in the development of resistance to this antimicrobial in this study. Therefore, in this resourceconstrained setting, the emergence of carbapenem resistance portends a bleak prospect for antimicrobial alternatives and access.

We did not observe any significant difference in the rate of multidrug resistance between ESBL-producers and nonESBL-producers. This discovery, however, is consistent with the fact that more than $90 \%$ of the isolates are multiply resistant. This multidrug resistance has made it harder to manage patients with infections caused by ESBL-producers, and it has raised the mortality rate of its victims [10], [34]. One limitation to our study is the fact that it was a laboratorybased design, with no patient contact, and therefore limited in clinical scope. Although, the clinical implications of our findings cannot be clearly determined, nevertheless, the study shows relatively high level of ESBL-production $(\sim 31 \%)$ among clinical GNB isolates in our hospital.

In conclusion, this study shows a high prevalence of ESBL-production among GNB involved in clinical infections in our hospital. The spread of these bacteria limit treatment options to the carbapenems to which a steady rise of resistance is being reported. Albeit, they remain the drugs of choice for treating infections caused by ESBLs producers. There is the need for the clinical microbiology laboratories to routinely include ESBLs detection methods for continuous surveillance of multidrug-resistant isolates and antibiograms to guide empirical therapy.

\section{ACKNOWLEDGMENT}

We acknowledge the staff of the Microbiology Laboratory of OAUTHC for their cooperation.

\section{REFERENCES}

[1] Y. Doi, R.A. Bonomo, D.C. Hooper, K.S. Kaye, J.R. Johnson, C.J Clancy, J.T. Thaden, M.E. Stryjewski, and D. van Duin: "GramNegative Bacterial Infections: Research Priorities, Accomplishments, and Future Directions of the Antibacterial Resistance Leadership Group.” Clin. Infect. Dis, 64(1):30-S35,2017. doi:10.1093/cid/ciw829.

[2] B.T. Tadesse, E.A. Ashley, S. Ongarello, J. Havumaki, M Wijegoonewardena, I.J. González, and S. Dittrich, "Antimicrobial 
resistance in Africa: a systematic review.” BMC Infect Dis, 17 (1): 616, 2017. doi:10.1186/s12879-017-2713-1.

[3] M. Bassetti, M. Peghin, A. Vena, and D.R. Giacobbe. "Treatment of Infections Due to MDR Gram-Negative Bacteria.” Front. Med, 6:74, 2019. doi:10.3389/fmed.2019.00074.

[4] D. Rawat and D. Nair, "Extended-spectrum $\beta$-lactamases in Gram Negative Bacteria." J Glob Infect Dis, 2(3): 263-274, 2010. doi:10/fktqxj.

[5] R.H.-P. Dhillon and J. Clark: "ESBLs: A Clear and Present Danger?" Crit Care Res Pract, 2012: 1-11, 2012. doi:10/cm2wj2.

[6] K.W. Benner, P. Prabhakaran, and A.S. Lowros, "Epidemiology of Infections Due to Extended-Spectrum Beta-Lactamase-Producing Bacteria in a Pediatric Intensive Care Unit.” J Pediatr Pharmacol Ther, 19 (2): 83-90, 2014. doi:10/gg3qqg.

[7] F.J. Giwa, O.T. Ige, D.M. Haruna, Y. Yaqub, T.Z. Lamido, and S.Y. Usman, "Extended - Spectrum Beta - lactamase Production and Antimicrobial Susceptibility Pattern of Uropathogens in a Tertiary Hospital in Northwestern Nigeria." Ann Trop Pathol, 9: 11-16, 2018. doi:10.4103/atp.atp.

[8] O.A. Aboderin, O. Adefehinti, B.W. Odetoyin, A.A. Olotu, I.N. Okeke, and O. Adeodu: "Prolonged febrile illness due to CTX-M-15 extendedspectrum $\beta$-lactamase-producing Klebsiella pneumoniae infection in Nigeria." Afr J Lab Med, 1(1), 2011. doi:10/gffeng.

[9] J.D.D. Pitout, P. Nordmann, K.B. Laupland, and L. Poirel, "Emergence of Enterobacteriaceae producing extended-spectrum $\beta$-lactamases (ESBLs) in the community" J Antimicrob Chemother, 56 (1): 52-59, 2005. doi:10/d4qsfg.

[10] R. Ben-Ami, J. Rodríguez-Baño, H. Arslan, J.D.D. Pitout, C. Quentin, E.S. Calbo, Ö.K. Azap, C. Arpin, A. Pascual, D.M. Livermore, J. Garau, and Y. Carmeli, "A Multinational Survey of Risk Factors for Infection with Extended-Spectrum $\quad \beta$-Lactamase-Producing Enterobacteriaceae in Non-hospitalized Patients." Clin Infect Dis, 49(5): 682-690, 2009. doi:10/d6t5v3.

[11] CLSI, "Performance Standards for Antimicrobial Susceptibility Testing," (2016).

[12] A.-P. Magiorakos, A. Srinivasan, R.B. Carey, Y. Carmeli, M.E. Falagas, C.G. Giske, S. Harbarth, J.F. Hindler, G. Kahlmeter, B. Olsson-Liljequist, D.L. Paterson, L.B. Rice, J. Stelling, M.J. Struelens, A. Vatopoulos, J.T. Weber, and D.L. Monnet, "Multidrug-resistant, extensively drug-resistant and pandrug-resistant bacteria: an international expert proposal for interim standard definitions for acquired resistance.” Clin Microbiol Infect Dis, 18 (3): 268-281, 2012. doi:10.1111/j.1469-0691.2011.03570.x.

[13] R Core Team, "R: A language and environment for statistical computing." 2019.

[14] M.S. Berends, C.F. Luz, A.W. Friedrich, B.N.M. Sinha, C.J. Albers, and C. Glasner, "AMR - An R Package for Working with Antimicrobial Resistance Data." Microbiology, 2019. doi:10.1101/810622;http://web.archive.org/web/2020070210140 0/https://www.biorxiv.org/content/10.1101/810622v3.

[15] F.E. Al-Otaibi, E.E. Bukhari, M. Badr, and A.A. Alrabiaa: "Prevalence and risk factors of Gram-negative bacilli causing blood stream infection in patients with malignancy." Saudi Med J, 37(9): 979-984, 2016. doi:10/f85jqc.

[16] A.Y. Peleg and H. Dc, "Hospital-Acquired Infections Due to GramNegative Bacteria." NEngl J Med, 362:1804-13, 2010. doi:10/bj34wz.

[17] B.W. Odetoyin, O. Olaniran, D.O. Afolayan, I.A. Aderibigbe, O. Alaka, and A.A. Onanuga, "Asymptomatic bacteriuria in an apparently healthy population and its relation to hypertension." Afr J Clin Exp Microbiol, 19 (4): 282, 2018. doi:10/gffef6.

[18] E.U. Israel, E.G. Sylvester, and A. Elijah, "The Use of Antibiotics in a Nigerian Tertiary Health Care Facility," 1(3): 25-31, 2015.

[19] O. Ouchar Mahamat, A. Tidjani, M. Lounnas, M. Hide, J. Benavides, C. Somasse, A.-S. Ouedraogo, S. Sanou, C. Carrière, A.-L. Bañuls, H. Jean-Pierre, Y. Dumont, and S. Godreuil, "Fecal carriage of extendedspectrum $\beta$-lactamase-producing Enterobacteriaceae in hospital and community settings in Chad." Antimicrob Resist Infect Control, 8(1): 169, 2019. doi:10/gg3tvq.

[20] N. Rahamathullah, S.K. Ahamed Khan, Z. Arshad Khan, A. Farrukh, H.M. Bashir, and A. Ahmad, "Prevalence of extended spectrum betalactamase producing Enterobacteriaceae in urine samples from Thumbay hospitals, U.A.E." Access Microbiol, 1(1A): 434, 2019. doi:10.1099/acmi.ac2019.po0254.

[21] P. Sawatwong, P. Sapchookul, T. Whistler, C.J. Gregory, O. Sangwichian, S. Makprasert, P. Jorakate, P. Srisaengchai, S. Thamthitiwat, C. Promkong, P. Nanvatthanachod, M. Vanaporn, and J. Rhodes, "High Burden of Extended-Spectrum $\beta$-Lactamase-Producing Escherichia coli and Klebsiella pneumoniae Bacteremia in Older Adults: A Seven-Year Study in Two Rural Thai Provinces.” Am J Trop Med Hyg, 100 (4): 943-951, 2019. 100:943-51, doi:10/gg3tvr.
[22] B.M. Musa, H. Imam, A. Lendel, I. Abdulkadir, H.S. Gumi, M.H. Aliyu, and A.G. Habib: "The burden of extended-spectrum $\beta$ lactamase-producing Enterobacteriaceae in Nigeria: a systematic review and meta-analysis.” Trans. R. Soc. Trop. Med. Hyg, 114(4), 241-248, 2020. doi:10/gg3trs.

[23] E.K. Oladipo, J.O. Ogunsola, B.S. Akinade, and E.H. Awoyelu: "Resistance of Clinical Isolates to Generation of Cephalosporins in a Tertiary Hospital in Ogbomoso, South-Western Nigeria." Res J Microbiol, 10 (2): 76-82, 201, 2015. doi:10.3923/jm.2015.76.82.

[24] J. Dantas Palmeira and H.M.N. Ferreira, "Extended-spectrum betalactamase (ESBL)-producing Enterobacteriaceae in cattle production a threat around the world." Heliyon, 6 (1): e03206, 2020. doi:10/gg3trx.

[25] J. Rodríguez-Baño, B. Gutiérrez-Gutiérrez, I. Machuca, and A. Pascual, "Treatment of Infections Caused by Extended-Spectrum-BetaLactamase-, AmpC-, and Carbapenemase-Producing Enterobacteriaceae." Clin Microbiol Rev, 31(2): e00079-17, 2018. doi:10.1128/CMR.00079-17.

[26] D. Kumar, A.K. Singh, M.R. Ali, and Y. Chander, "Antimicrobial Susceptibility Profile of Extended Spectrum $\beta$-Lactamase (ESBL) Producing Escherichia coli from Various Clinical Samples." Infect Dis, 7: 1-8, 2014. doi:10.4137/IDRT.S13820.

[27] S. Vemula and R. Vadde, "Prevalence of ESBL-Producing Klebsiella pneumoniae Isolates in Tertiary Care Hospital." ISRN Microbiol, 2011: 318-48, 2011.

[28] A.-S. Ouédraogo, S. Sanou, A. Kissou, A. Poda, S. Aberkane, N. Bouzinbi, B. Nacro, R. Ouédraogo, P. Van De Perre, C. Carriere, D. Decré, H. Jean-Pierre, and S. Godreuil, "Fecal Carriage of Enterobacteriaceae Producing Extended-Spectrum Beta-Lactamases in Hospitalized Patients and Healthy Community Volunteers in Burkina Faso." Microb Drug Resist, 23 (1): 63-70, 2017. doi:10/f9k998.

[29] D.S. Teklu, A.A. Negeri, M.H. Legese, T.L. Bedada, H.K. Woldemariam, and K.D. Tullu, "Extended-spectrum beta-lactamase production and multi-drug resistance among Enterobacteriaceae isolated in Addis Ababa, Ethiopia." Antimicrob Resist Infect Control, 8 (1): 39, 2019. doi:10.1186/s13756-019-0488-4.

[30] Y. Mohammed, G.B. Gadzama, S.B. Zailani, and A.O. Aboderin, "Characterization of extended-spectrum beta-lactamase from Escherichia coli and Klebsiella species from North Eastern Nigeria." J Clin Diagn Res, JCDR. 10 (2): DC07, 2016.

[31] H. Ogefere, P. Aigbiremwen, and R. Omoregie, "Extended-Spectrum Beta-Lactamase (ESBL)-Producing Gram-negative Isolates from Urine and Wound Specimens in a Tertiary Health Facility in Southern Nigeria.” Trop J Pharm Res, 14 (6): 1089, 2015. doi:10/gffcpv.

[32] F.S. Codjoe and E.S. Donkor, "Carbapenem Resistance: A Review." Med Sci, 6 (1): 2017. doi:10/c9zd.

[33] Z.D. Pana and T. Zaoutis, "Treatment of extended-spectrum $\beta$ lactamase-producing Enterobacteriaceae (ESBLs) infections: what have we learned until now?" F1000Research, 7, 2018. doi:10.12688/f1000research.14822.1. 